\title{
A Fundamental Time-Domain and Linearized Eigenvalue Analysis of Coalesced Power Transmission and Unbalanced Distribution Grids using Modelica and the OpenIPSL
}

\author{
Marcelo de C. Fernandes ${ }^{1, \dagger} \quad$ Luigi Vanfretti ${ }^{1, \ddagger} \quad$ Janaína G. de Oliveira ${ }^{2} \quad$ Maxime Baudette $^{3}$ \\ ${ }^{1}$ Dept. of Electrical, Computer and Systems Engineering, Rensselaer Polytechnic Institute, USA,

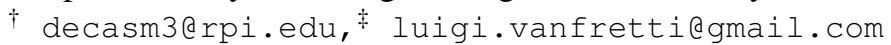 \\ ${ }^{2}$ Dept. of Electrical Energy, Federal University of Juiz de Fora, Brazil, janaina. oliveira@ufjf .edu.br \\ ${ }^{3}$ Grid Integration Group, Energy Storage \& Distributed Resources Division, Lawrence Berkeley National Laboratory, \\ Berkeley, CA, USA, baudette@lbl.gov
}

\begin{abstract}
This paper present mathematical modeling and implementation in Modelica language of a coalesced electric power transmission and distribution system model. To this end, a newly developed feature in OpenIPSL that allows to amalgamate power transmission and distribution networks at the equation level is described, two different sample power systems are assembled and three simulations are performed for each of them in a Modelica-compliant software. Dynamic simulations are carried out to perform comparisons between different modeling approaches for a distribution feeder and among different load characteristics. Moreover, each simulation is linearized using a script in ten specific time instants and an eigenvalue comparison is performed. Results show that the conventional positive sequence models may lead to errors about the dynamic behavior of the entire system, specially when considering unbalances in distribution networks.

Keywords: Modelica, Power Systems, Hybrid Models, Linearization, Eigenvalues, Transmission Networks, Distribution Networks
\end{abstract}

\section{Introduction}

\subsection{Paper Motivation}

In the past few years, the world has began to undergo an important energy transition. Major environmental concerns and the need for diversify the energy mix have pushed society to look for alternative energy sources. As a consequence, governments have encouraged investments in renewable energy in order to increase energy system sustainability.

This energy transition has a major importance in the electric power sector, in which renewable sources are being integrated. Renewable technologies accounted for $25 \%$ of the world's generation of electricity in 2016 (IEA., 2017). In addition, renewable energy supply has increased 4\% a year since 2000 (IEA., 2017), showing that the share of renewable energy sources in power systems will not slowdown in the near future.
Renewable energy sources may be connected to the power grid on medium or low voltage levels as Distributed Generation (DG), bringing challenges to the grid's operation (Boemer et al., 2011). These challenges are a consequence of the lack of understanding and adequate modeling of Distributed Energy Resources (DERs) during the design phase, particularly their control and protection, in studies of their performance when integrated to transmission grids (ENTSO-E, 2014).

Therefore, it has become evident that distribution networks, and all its components, can no longer be neglected from studies assessing power systems and its dynamic behavior (Jain et al., 2016). In order to address these issues, many tools and strategies have been proposed in the last years in order to perform the analysis of joint power transmission and distribution (T\&D) systems. These tools, their modeling strategies and simulation approaches are discussed next.

\subsection{Background}

Modeling assumptions established ever since the beginning of long distance $\mathrm{AC}$ power transmission in the early 1900 's have led to a decoupled treatment of transmission from distribution, and vice versa. Thus, analysis of each of them has been carried out individually, using a different approach for each network in order to analyze their behavior. As an example, the transmission system is often represented on its single-phase equivalent or positive sequence modeling (Tinney and Hart, 1967). This is due to the assumption that bulk power systems can be considered to operate under completely balanced conditions. On the other hand, distribution grids normally undergo unbalances due to many factors, and thus, simulation tools for distribution networks commonly used three-phase models (Garcia et al., 2000).

In fact, many proprietary and commercial software packages made for the study of the power system are built upon the aforementioned assumptions, especially when the software provides phasor time-domain analysis routines. For instance, positive sequence modeling is typi- 
cal in software packages such as PSS/E (Siemens) originally developed in the U.S.A., Anarede (Cepel, a) and Anatem (Cepel, b) developed in Brazil. On the other hand, three-phase analysis methods are available in CYMDIST (EATON) and OpenDSS (EPRI) both developed in North America. Coupling between models from these type of tools is generally only possible through co-simulation and the common belief of practitioners was that these modeling approaches were largely incompatible (Balasubramaniam and Abhyankar, 2017).

In order to solve this assumed incompatibility between modeling approaches and simulation tools, a hybrid model, named Monotri, was proposed in (Marinho and Taranto, 2008). This model was the first to provide a "physical interface" between a system modeled in positive sequence with another one using three-phase modeling, for power flow studies. Concurrently, the same research group highlighted the importance of considering DERs in dynamic simulations (Assis et al., 2006). Later, the hybrid formulation presented in (Marinho and Taranto, 2008) was extended in (Taranto and Marinho, 2017) to transient stability studies, i.e. dynamic modeling and simulation. This hybrid model has two main benefits: (1) it provides a "physical interface" to couple Transmission and Distribution T\&D instead of using co-simulation, and (2) it combines usual modeling approaches that are familiar to power system domain users.

\subsection{Modelica Tools for Power Systems}

Previous work has shown that the Modelica language is a promising alternative for modeling the complexity of modern power systems (Vanfretti et al., 2013). For instance, (Mirz et al., 2016) present a multi-level approach to model power electronics in power systems using Modelica, while (Casella et al., 2016, 2017) study the feasibility of using Modelica-based tools to solve large power system models.

Along these and other many studies, developers have built several Modelica libraries for power system simulation. The study presented in (Winkler, 2017) lists many libraries for power system analysis, along with their history, modeling principles, library structure, weaknesses and strengths. Among those packages there is the OpenIPSL (Baudette et al., 2018).

According to (Winkler, 2017), the OpenIPSL package has many strengths such as its robustness and its models. OpenIPSL models underwent software-tosoftware validation against domain-specific proprietary and open source software packages including PSS/E and PSAT (Milano, 2005). In addition, OpenIPSL is friendly to users familiar to typical power system analysis, as it addresses resistance to change-factors, associated with the use of new technologies (Vanfretti et al., 2014). OpenIPSL is still being developed in voluntary basis as an Open Source Software project on Github (http:// openips l.org) and new features are being added. For example, its latest version comes with an application ex- ample package made for modeling positive sequence systems with three-phase networks using the hybrid interface proposed in (Marinho and Taranto, 2008), for which some results are reported in (de Castro Fernandes et al., 2018) (in Portuguese).

In addition, models built using the OpenIPSL package may take advantage of the rich features available in Modelica-based tools, such as linearization. Linear analysis and small-signal studies for integrated T\&D systems are not commonly available in power system tools. However, this analysis can be extremely useful to understand the dynamics of DERs at lower voltage levels. With Modelica tools it is possible to perform this analysis without the need of encoding the linear and non-linear models separately. This paper exploits and demonstrates this possibility, by analyzing a hybrid positive-sequence and threephase T\&D model implemented using OpenIPSL.

\subsection{Paper Contributions}

The main contributions from this paper are listed below:

- Model description and implementation in the Modelica language of a hybrid single-phase $x$ threephase element, $\pi$-modeled three-phase lines and wye-grounded loads. All using a phasor approach with an OpenIPSL library.

- Exploring the rich features of Modelica language for simulation, linearization and eigenvalue analysis of a small joint-modeled transmission and distribution system, avoiding the co-simulation approach.

- Analyses and comparisons of the positive-sequence versus the hybrid-modeling approach in the stability analysis of power systems.

\section{Mathematical Modeling}

\subsection{Studied Power Network}

The power system studied in this paper is described in Figure 1 and it consists of an adaptation of IEEE 14-bus test system, first implemented using OpenIPSL in (Murad et al., 2015). This transmission network was extended to include synchronous machines and a distribution feeder.

To model different modeling impacts of unbalanced distribution networks, loads at buses 2 and 11 are modified, one at a time, in different case studies. The modification is based upon an extension of the original load itself, to include a distribution feeder consisting of two buses, a power line and a load. In one set of tests, the load on bus 2 is replaced by this distribution feeder and in another set of tests, the replaced load is on bus 11. The distribution feeder is connected to the respective bus by a transformer. This element must be modeled using the hybrid formulation proposed in (Marinho and Taranto, 2008). The remainder of the power system is modeled using positivesequence models, including all dynamic components as specified in (Kodsi and Canizares, 2003), starting from 
the implementation in (Murad et al., 2015). The generators are labeled $G 1$ and $G 2$, while condensers are $C 1, C 2$ and $C 3$, in Figure 1.

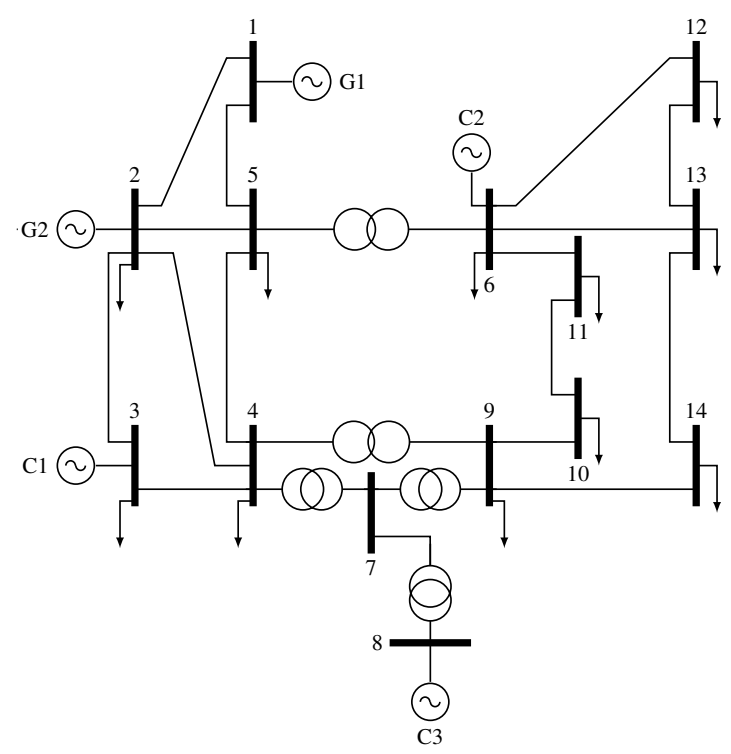

Figure 1. Modified IEEE 14-bus test system diagram.

Positive-sequence buses and branches are available in the OpenIPSL library while the hybrid and three-phase elements are included in the . ApplicationExamples.ThreePhase package, within the OpenIPSL distribution. Models for all machines are also available in the OpenIPSL library. The ThreePhase package was recently added by the authors (Baudette et al., 2018; de Castro Fernandes et al., 2018) and thus, it is useful to present the mathematical modeling of such elements, along with their implementation in the Modelica language.

\subsection{Hybrid and Three-Phase Models}

This subsection introduces the mathematical formulation for hybrid and three-phase models. It is important to note that since this paper is related to power system modeling, the value for each variable is represented in per unit ( $p$. u.), which is the representation of the system's quantities as fraction of a base value. For this subsection, values of voltage, current, power, admittance and conductance, are represented in this way. In addition, variables written in bold are matrices and vectors while variables written in regular letters represent scalars. In addition, variables with the upper bar $\left(^{-}\right)$denotes phasors.

The formulation of a hybrid power component is proposed in (Marinho and Taranto, 2008; Taranto and Marinho, 2017) and it consists of a passive $\pi$ element, as depicted in Figure 2.

Equations for a three-phase $\pi$ element are:

$$
\left[\begin{array}{l}
\mathbf{I}_{k}^{a b c} \\
\mathbf{I}_{m}^{a b c}
\end{array}\right]=\left[\begin{array}{cc}
\mathbf{Y}_{s e r}^{a b c}+\mathbf{Y}_{s h t_{k}}^{a b c} & -\mathbf{Y}_{s h t_{k}}^{a b c} \\
-\mathbf{Y}_{s e r}^{a b c} & \mathbf{Y}_{s e r}^{a b c}+\mathbf{Y}_{s h t_{m}}^{a b c}
\end{array}\right]\left[\begin{array}{c}
\mathbf{V}_{k}^{a b c} \\
\mathbf{V}_{m}^{a b c}
\end{array}\right]
$$

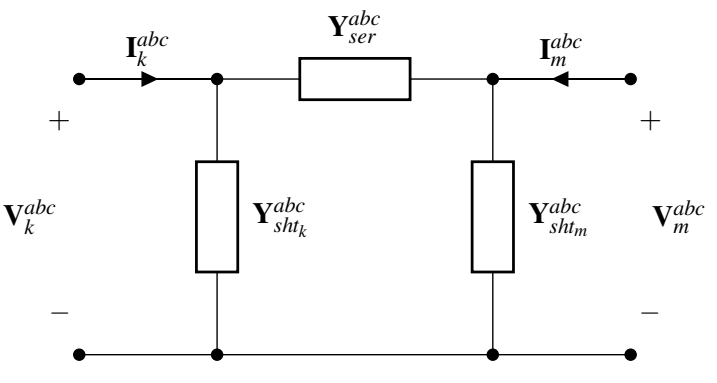

Figure 2. Representation of three-phase, passive $\pi$-equivalent component, such as a distribution power line or transformer.

where $\mathbf{V}_{x}^{a b c}, \bar{I}_{x}^{a b c}$ are vectors of three-phase voltage and injected current phasors, respectively, for a terminal $x=$ $k, m$, while $\mathbf{Y}_{s e r}^{a b c}$ and $\mathbf{Y}_{s h t}^{a b c}$ are series and shunt admittance matrices, respectively.

Assuming that the system in terminal $k$ is completely balanced and that there is no negative component current source in terminal $m$, it is possible to ignore zero and negative sequence components, resulting in:

$$
\left\{\begin{aligned}
\mathbf{V}_{k}^{a b c}=\mathbf{T}_{1} \bar{V}_{k}^{+}=\left[\begin{array}{c}
1 \\
\alpha^{2} \\
\alpha
\end{array}\right] \bar{V}_{k}^{+} \\
\bar{I}_{k}^{+}=\mathbf{T}_{2} \mathbf{I}_{k}^{a b c}=\frac{1}{3}\left[\begin{array}{lll}
1 & \alpha & \alpha^{2}
\end{array}\right] \mathbf{I}_{k}^{a b c}
\end{aligned}\right.
$$

where $\alpha=e^{j \frac{2 \pi}{3}}$ and $\bar{V}_{k}^{+}$e $\bar{I}_{k}^{+}$are positive-sequence phasors for voltage and injected current in terminal $k$, respectively. Therefore, replacing (2) in (1) it is possible to write the final equation for a hybrid model:

$$
\left[\begin{array}{c}
\bar{I}_{k}^{+} \\
\mathbf{I}_{m}^{a b c}
\end{array}\right]=\left[\begin{array}{ll}
\mathbf{M}_{S S} & \mathbf{M}_{S T} \\
\mathbf{M}_{T S} & \mathbf{M}_{T T}
\end{array}\right]\left[\begin{array}{c}
\bar{V}_{k}^{+} \\
\mathbf{V}_{m}^{a b c}
\end{array}\right]
$$

where

$$
\begin{aligned}
\mathbf{M}_{S S} & =\mathbf{T}_{2}\left(\mathbf{Y}_{s e r}^{a b c}+\mathbf{Y}_{s h t_{k}}^{a b c}\right) \mathbf{T}_{1} \\
\mathbf{M}_{S T} & =\mathbf{T}_{2}\left(-\mathbf{Y}_{s e r}^{a b c}\right) \\
\mathbf{M}_{T S} & =\left(-\mathbf{Y}_{s e r}^{a b c}\right) \mathbf{T}_{1} \\
\mathbf{M}_{T T} & =\mathbf{Y}_{s e r}^{a b c}+\mathbf{Y}_{s h t_{m}}^{a b c}
\end{aligned}
$$

A transmission or distribution power line can also be modeled as a three-phase passive $\pi$ element (Arrillaga et al., 2001) and can also be described by (1). This model is adequate to represent unbalanced transmission lines such as the ones encountered in power distribution systems.

The model of the a three-phase load with grounded-wye connection is depicted in Figure 3. This load model will 


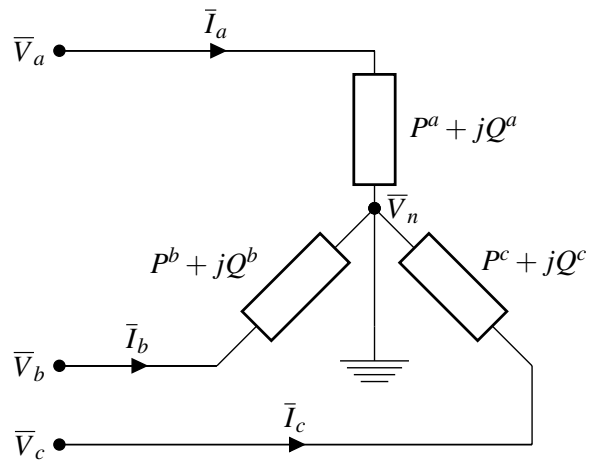

Figure 3. Representation of a generic three-phase load in a grounded wye connection.

be considered for the tests when the distribution feeder has a three-phase representation. For this load model, it is possible to write one equation for each phase independently:

$$
\left\{\begin{array}{l}
\bar{S}_{a}=P_{a}+j Q_{a}=\bar{V}_{a n} \bar{I}_{a}^{*} \\
\bar{S}_{b}=P_{b}+j Q_{b}=\bar{V}_{b n} \bar{I}_{b}^{*} \\
\bar{S}_{c}=P_{c}+j Q_{c}=\bar{V}_{c n} \bar{I}_{c}^{*}
\end{array}\right.
$$

To define the active and reactive power on each phase ( $P_{x}$ and $Q_{x}$ ), the ZIP load model (Kersting, 2001) was chosen. It allows the representation of a load as a composition of three types of characteristics: constant power, constant current and constant impedance. In addition, it shows how the power demanded by the load varies according to its terminal voltage $V_{x n}$. Therefore, it is possible to write (6) for each phase, represented in the equation by under-script $x$. The load demands $P_{0}$ and $Q_{0}$ are those for a terminal voltage of $V_{x n}^{0}$. The coefficient $\alpha$ shows how much of the load is constant power (superscript ${ }^{p}$ ), current (superscript ${ }^{i}$ ) or impedance (superscript ${ }^{z}$ ).

$$
\left\{\begin{array}{l}
P_{x}\left(V_{x n}\right)=P_{x}^{0}\left[\alpha_{x}^{p}+\alpha_{x}^{i}\left(\frac{V_{x n}}{V_{x n}^{0}}\right)+\alpha_{x}^{z}\left(\frac{V_{x n}}{V_{x n}^{0}}\right)^{2}\right] \\
Q_{x}\left(V_{x n}\right)=Q_{x}^{0}\left[\alpha_{x}^{p}+\alpha_{x}^{i}\left(\frac{V_{x n}}{V_{x n}^{0}}\right)+\alpha_{x}^{z}\left(\frac{V_{x n}}{V_{x n}^{0}}\right)^{2}\right] \\
\alpha_{x}^{p}+\alpha_{x}^{i}+\alpha_{x}^{z}=1
\end{array}\right.
$$

\section{Modelica Implementation}

This section describes how the models developed in Section 2 were written in Modelica, and implemented within the OpenIPSL package. Because the actual implementation of each new component is rather large, this section aims to illustrate how the models were implemented, and not to document the implementation itself.

\subsection{Studied Power Network in OpenIPSL}

The IEEE 14-bus test system implemented in Modelica using OpenIPSL models is illustrated in Figure 4. The blue line shows the connection between elements. These connections are made between $\mathrm{PwP}$ ins from different models. The PwPin is the main feature upon which the connections of electrical components is made. The pin itself works as an electrical circuit node allowing the flow of complex current variables, and providing two important pieces of information (supposing a $\mathrm{PWP}$ in named $\mathrm{P}$ ):

- real and imaginary part of the voltage at that particular node (P.Vr and P.vi);

- real and imaginary part of the current flowing through that node (P.ir and P.ii).

In a single-phase equivalent model, the pin has positive sequence voltages and currents. On the other hand, in a three-phase model, one pin is necessary for each phase represented. The connection between the machine G1 (namely GenBus1 in Figure 4) to bus B1 is defined using one-line code, as follows:

connect (GenBus1.pwPin, B1.p) ;

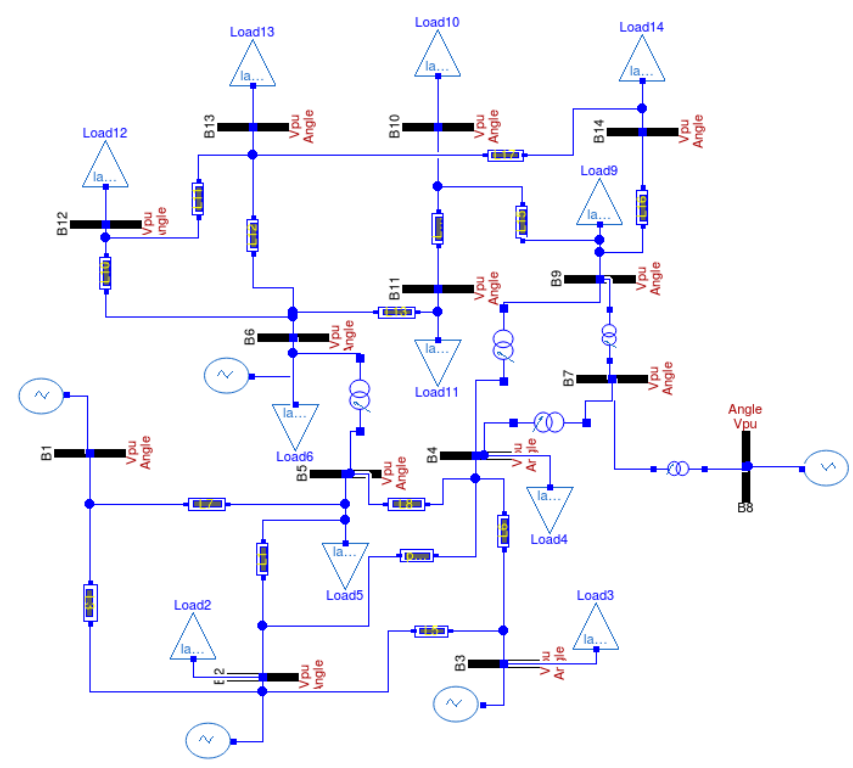

Figure 4. Original IEEE 14-bus system implemented in a Modelica-compliant software using OpenIPSL.

However, for the connection between the groundedwye connected three-phase load and three-phase bus Bus632, three lines of code are needed:

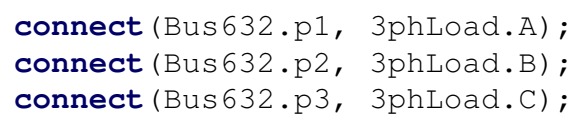

It is important to note that a pin representing phase $\mathrm{A}$ in one component must always be connected with respective pin representing the same phase in another component. 


\subsection{Hybrid Transformer}

The element that interfaces the positive-sequence model with the three-phase distribution feeder is a transformer in a $\Delta-Y$ connection. This connection is chosen for consistency with the equations (2). In this case, a function TransformerFCn. D_Yg was implemented and is imported into the model in order to provide the values of $M_{S S}, M_{S T}, M_{T S}, M_{T T}$ from (3) for a transformer in a $\Delta-Y$ connection. Calculating these matrices with functions is the most efficient approach because each transformer connection has a different $Y_{s e r}^{a b c}, Y_{s h t, k}^{a b c}$ and $Y_{s h t, m}^{a b c}$. This helps in describing, the hybrid transformer model as implemented below. The values for reactance and resistance are only used for illustration.

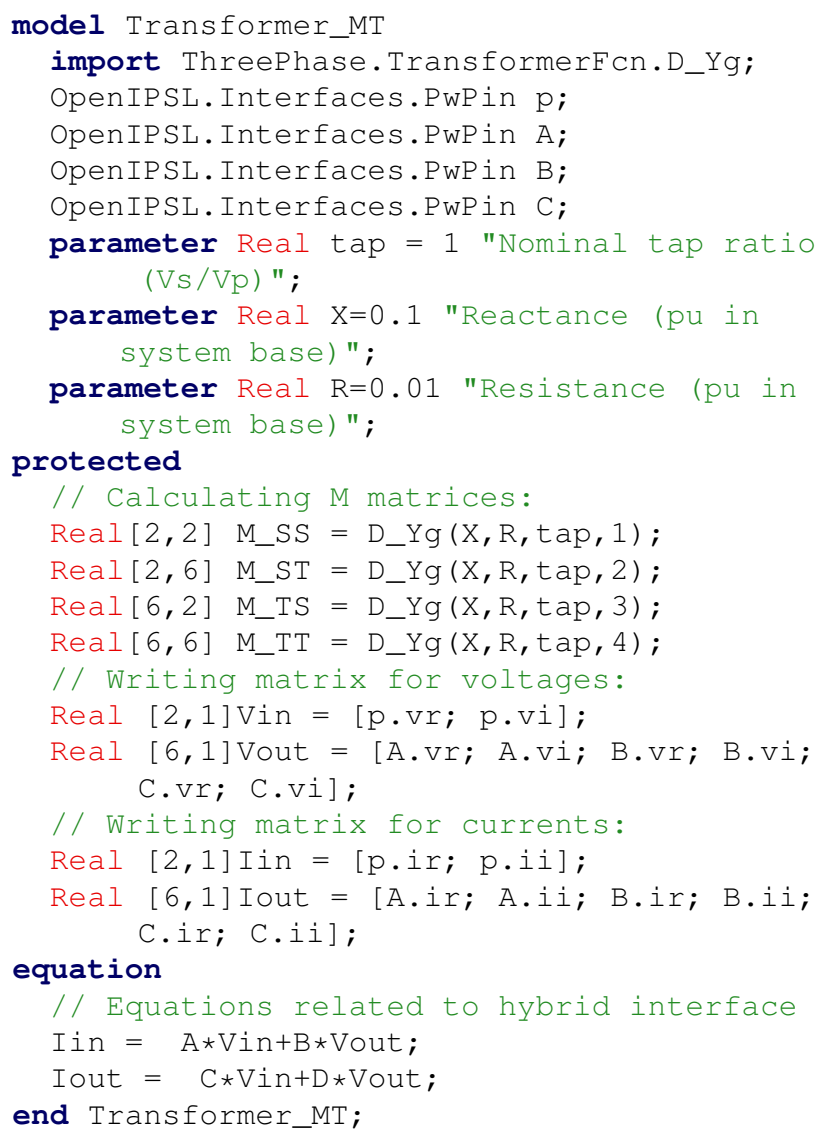

\subsection{Three-Phase Line}

The implementation of the three-phase transmission line modeled as a $\pi$-element is straight forward using Modelica. However, observe that due to the PwP in voltage and current convention, complex numbers are not used but instead two real numbers representing imaginary and real parts are used in the PwPin. Therefore, matrices $Y_{s e r}^{a b c}$ and $Y_{\text {sht }}^{a b c}$ are designed according to this convention. Using equation (1) it is possible to write the following model:

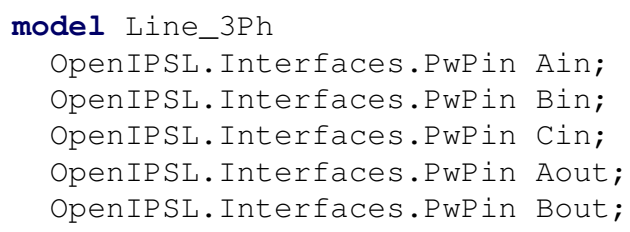

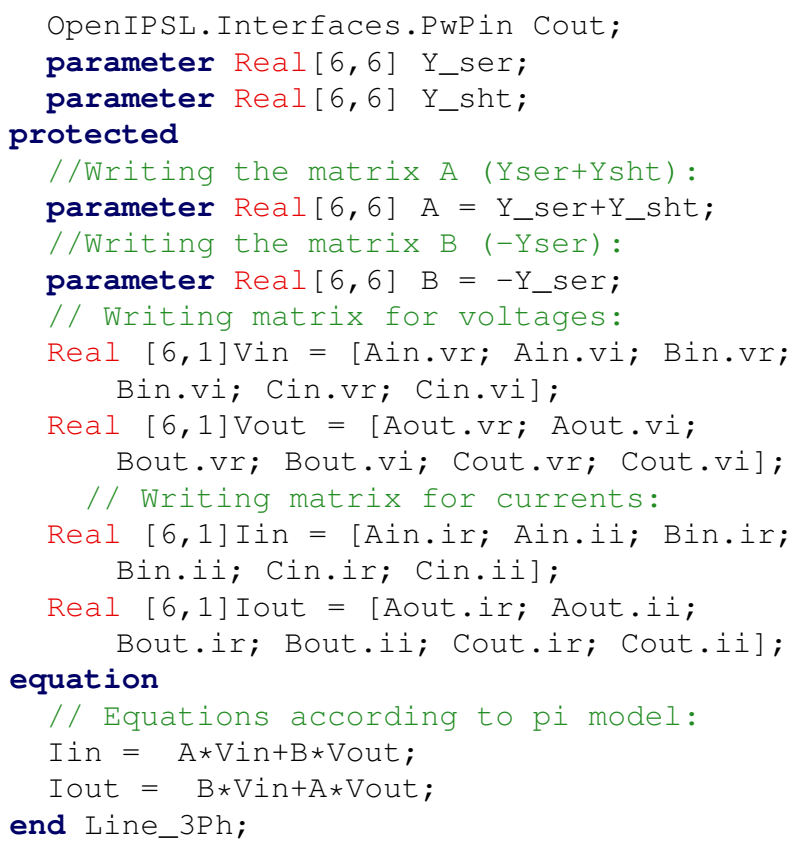

\subsection{Three-Phase Load}

The three-phase load model implementation in Modelica is also straight forward. The implementation of equation (6) for each phase is shown in the Modelica code below. The code shows the example values for active and reactive power. In addition, note that a constant impedance load is being used.

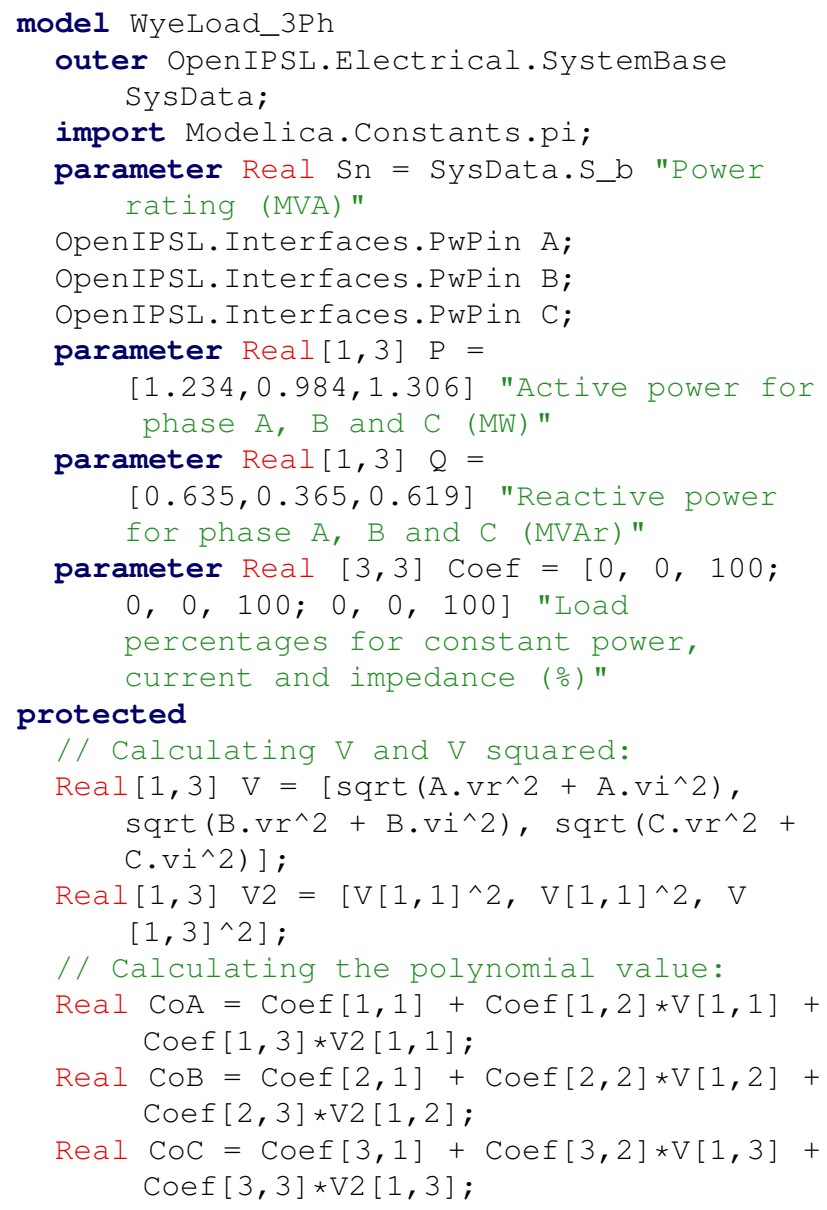




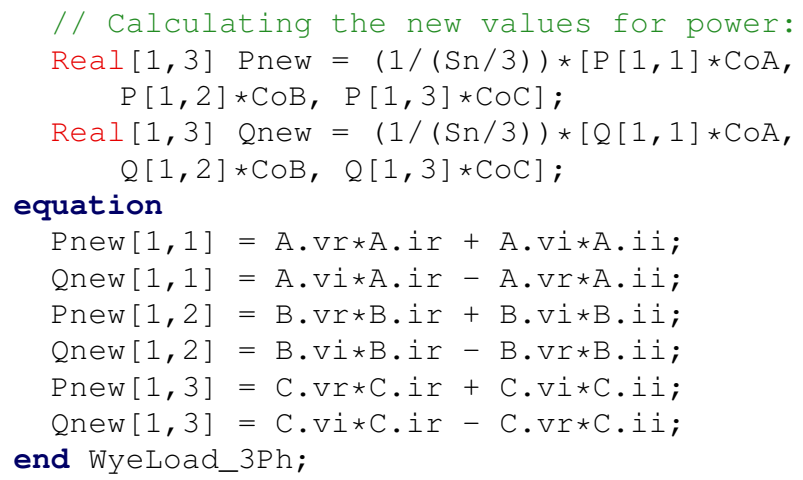

\section{Simulation Methodology}

In this paper, the original IEEE 14-bus system, depicted in Figure 4, is modified in two different ways and three simulations are performed in each modified system, resulting in a total of six dynamic simulations. The common characteristic among all six experiments is that a small feeder, consisting of two buses, one transformer, one transmission line and one load is added to a transmission bus, effectively extending it to represent a T\&D system. In three experiments this feeder replaces the load in bus number 11. In the remaining three experiments, the feeder replaces the load in bus number 02 .

Consider the experiments related to the feeder addition to bus 02 . One experiment will consist of the addition of a feeder modeled in positive sequence, as depicted in Figure 5(a). The load at the end of the feeder increase for 9 times, every 12 seconds. In the second experiment, the feeder is modeled in three-phase and the connection is made by a hybrid transformer, shown in Figure 5(b). In that experiment, the load increase occurs in a balanced way, again 9 times and every 12 seconds. The last experiment for bus 02 is based in the addition of the same three-phase feeder depicted in Figure 5(b), however, the load increases with phase unbalances.

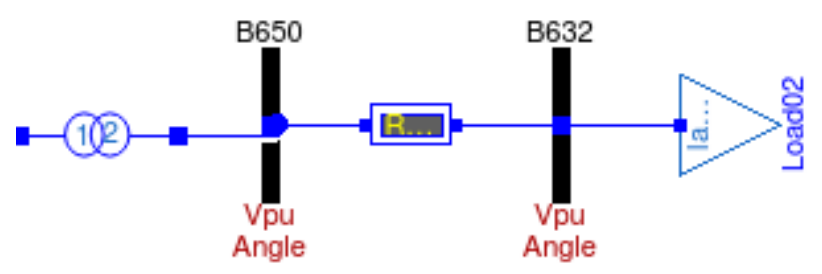

(a) Positive sequence model.

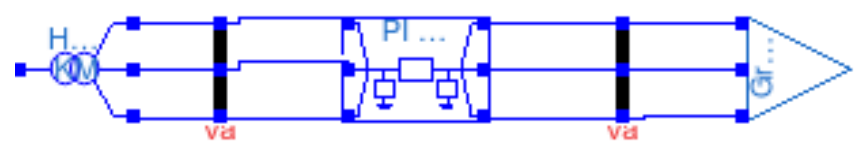

(b) Three-phase model.

Figure 5. Distribution feeder model diagrams used in the experiments.
The experiments related to the addition of the feeder to bus 11 are similar to what was described for bus 02 . The difference between both set of experiments lies in parameter values for the line, load and transformer. All simulations are listed in Table 1.

Table 1. Summary of simulations.

\begin{tabular}{cl}
\hline Test & Description \\
\hline I & $\begin{array}{c}\text { Distribution feeder connected to bus 02. } \\
\text { Positive sequence model. } 9 \text { load steps }\end{array}$ \\
\hline II & $\begin{array}{c}\text { Distribution feeder connected to bus 02. } \\
\text { Three-phase model. } 9 \text { balanced load steps }\end{array}$ \\
\hline III & $\begin{array}{c}\text { Distribution feeder connected to bus 02. } \\
\text { Three-phase model. } 9 \text { unbalanced load steps }\end{array}$ \\
\hline IV & $\begin{array}{c}\text { Distribution feeder connected to bus } 11 . \\
\text { Positive sequence model. } 9 \text { load steps. }\end{array}$ \\
\hline V & $\begin{array}{c}\text { Distribution feeder connected to bus } 11 . \\
\text { Three-phase model. } 9 \text { balanced load steps. }\end{array}$ \\
\hline VI & $\begin{array}{c}\text { Distribution feeder connected to bus 11. } \\
\text { Three-phase model. } 9 \text { unbalanced load steps. }\end{array}$ \\
\hline
\end{tabular}

\section{System and Simulation Parameters}

This section provides information about the parameters used for the simulation of the system presented in Sections 2 and 3 and in Subsection 4. Table 2 lists the machine models that were used in this study. All machines, generators and condensers, are represented with the same dynamic model. The parameters for these machines along with voltage regulators are described in (Kodsi and Canizares, 2003), which also provides load values and branch parameters. In order to increase the total load of the system, the load values reported in the original IEEE14 system are multiplied by a factor of 1.4 , which is the same procedure adopted in (Kodsi and Canizares, 2003). It is very important to mention that the machines do not include automatic speed regulators. Thus, the value for mechanical power is kept constant during all the simulation and equal to the steady state value of electrical power in the initial condition. This means that with load increasing, the system's frequency will drift away from $60 \mathrm{~Hz}$ to lower values. However, in this performed study the frequency does not change significantly from the base value.

Table 2. Parameters for synchronous machines.

\begin{tabular}{ll}
\hline Equipment & Model used \\
\hline Machine & PSAT.Order6 \\
Voltage regulator & PSAT.AVR.AVRTypeII \\
Speed regulator & - \\
\hline
\end{tabular}

Table 3 lists system-wide parameters, such as its frequency and its base power. The latter is used to calculate 
all per-unit values in the system, such as the resistance and reactance from the hybrid transformer.

Table 3. System parameters.

\begin{tabular}{ll}
\hline Description & Value \\
\hline System Frequency & $60 \mathrm{~Hz}$ \\
Power Base & $100 \mathrm{MVA}$ \\
\hline
\end{tabular}

Table 4 presents all the simulation data chosen for running in all simulations (i.e. scenarios I to VI).

Table 4. Simulation parameters.

\begin{tabular}{ll}
\hline Description & Value \\
\hline Duration & $120 \mathrm{~s}$ \\
Interval Size & $0.005 \mathrm{~s}$ \\
Integration Method & dassl \\
Tolerance & $10^{-5}$ \\
\hline
\end{tabular}

The values for transformer's reactance $\left(X_{t f}\right)$, power line's mutual $\left(Z_{m}\right)$ and self $\left(Z_{s}\right)$ impedance, and load $\operatorname{steps}(\Delta S)$ are important parameters that need to be explicitly described. They are summarized in Table 5 .

\section{Simulation Results and Lineariza- tion Analysis}

All tests described in Section 4 were simulated using a Modelica-compliant software tool. Results for the simulations corresponding to the distribution feeder modeled in positive sequence (simulations I and IV) are shown in Figure 6. Bus 02 voltage in simulation I is depicted in Figure 6(a), while bus 11 voltage in simulation IV, is shown in Figure 6(b). Note that larger oscillations occur in simulation I, which are due to the value of the load step. However, the voltage variation, in per unit (pu), is higher in simulation IV than I. This is due to the location of the distribution feeder where the load is increased.

The comparison between bus 02 voltage in simulations I, II and III is shown in Figure 7(a), during the last load step increase, which occurs between $108 \mathrm{~s}$ and $120 \mathrm{~s}$. In that figure, the blue solid curve represents the voltage curve for simulation I, while the dashed red line, marked with squares, represents simulation II and the solid green line, marked with circles, represents simulation III. It is important to observe that the curve for simulation II overlaps the one for simulation I. This result should be expected because a perfect balanced three-phase system may be represented its positive sequence equivalent model, which implies that the three-phase implementation has been performed correctly.

The second important fact that should be observed is that the green curve, resulting from simulation III, has a different value if compared to the blue and red curves. The difference may be small (approximately $0.05 \%$ ) but it is important to be noted. This divergence between the curves should be expected, since the unbalance should reduce the value of positive sequence component.

A similar analysis is done in Figure 7(b), which depicts voltage in bus 11 for simulations IV, V and VI during the last load step increase. The solid blue line curve represents the voltage curve for simulation IV, the dashed red line, marked with squares, represents the voltage for simulation $\mathrm{V}$ and the solid green line, marked with circles, represents the result for simulation VI. Note that, again, blue and red curves overlaps, as expected, and the green curve diverges from the other two.

After verifying that the voltage curves behaves as expected in time domain simulations, a script was made in order to explore the easy access to linearization process provided by Modelica language. The script linearizes each of the six simulations one time step, $0.005 s$, before the load step increase, totaling the value of ten processes for every simulation. From each linear model, fifty eigenvalues are extracted and stored for comparison. As expected, the eigenvalues from the ten linear models coming from simulation I are virtually the same ones coming from simulation II. The total absolute error between the fifty eigenvalues from the ten linear models coming from simulations I and II is 0.00017 . The same fact is observed when comparing the eigenvalues from linear models coming from simulations IV and V. In this case, the total error between the eigenvalues is 0.00001 .

The next analysis conducted in this paper is made using specific eigenvalue collected from in simulations I and III. It is important to observe that both eigenvalues become apart from each other, and the distance increase with the load step. The difference between this eigenvalue locus is shown in Figure 8. Note that the eigenvalues from the unbalanced case have less negative real part and more negative imaginary part if compared to the respective eigenvalue from the balanced case.

A similar analysis is conducted using the comparison of one specific eigenvalue coming from simulations IV and VI. Again, the eigenvalues become more distant with the load variation. This variation is shown in Figure 9. In this case, the eigenvalue has no imaginary part but the real part of the eigenvalue become less negative with load steps.

\section{Discussion}

The distance between the eigenvalues from the different linearized systems is of great interest, because it provides crucial information about the system's stability. Here, the error in damping $(\Delta \sigma)$ and in angular frequency $(\Delta \omega)$ are analyzed. Table 6 presents the difference in damping and angular frequency in the eigenvalues represented in Figure 8. Note that the difference in both damping and frequency is not linear. Table 7 presents the difference in damping for eigenvalues depicted in Figure 9 and, again, the difference is not linear. 
A Fundamental Time-Domain and Linearized Eigenvalue Analysis of Coalesced Power Transmission and Unbalanced Distribution Grids using Modelica and the OpenIPSL

Table 5. Simulation system parameters details.

\begin{tabular}{|c|c|c|c|c|}
\hline Test & $X_{t f}(p u)$ & Line Data $(p u)$ & $\Delta S(p u)$ & Load Description \\
\hline $\begin{array}{l}\text { I } \\
\text { III }\end{array}$ & 0.11001 & $\begin{array}{l}Z_{m}=0.0032+j 0.0099 \\
Z_{s}=0.0226+j 0.0690\end{array}$ & $3.038+j 1.778$ & $\begin{array}{l}\text { Positive sequence increase. } \\
\text { Divided equally between phases A,B,C. } \\
\text { Divided equally between phases A and C. }\end{array}$ \\
\hline $\begin{array}{l}\text { IV } \\
\text { V } \\
\text { VI }\end{array}$ & 0.55618 & $\begin{array}{l}Z_{m}=0.0233+j 0.0494 \\
Z_{s}=0.2443+j 0.2493\end{array}$ & $0.490+j 0.252$ & $\begin{array}{l}\text { Positive sequence increase. } \\
\text { Divided equally between phases A,B,C. } \\
\text { Divided equally between phases A and C. }\end{array}$ \\
\hline
\end{tabular}

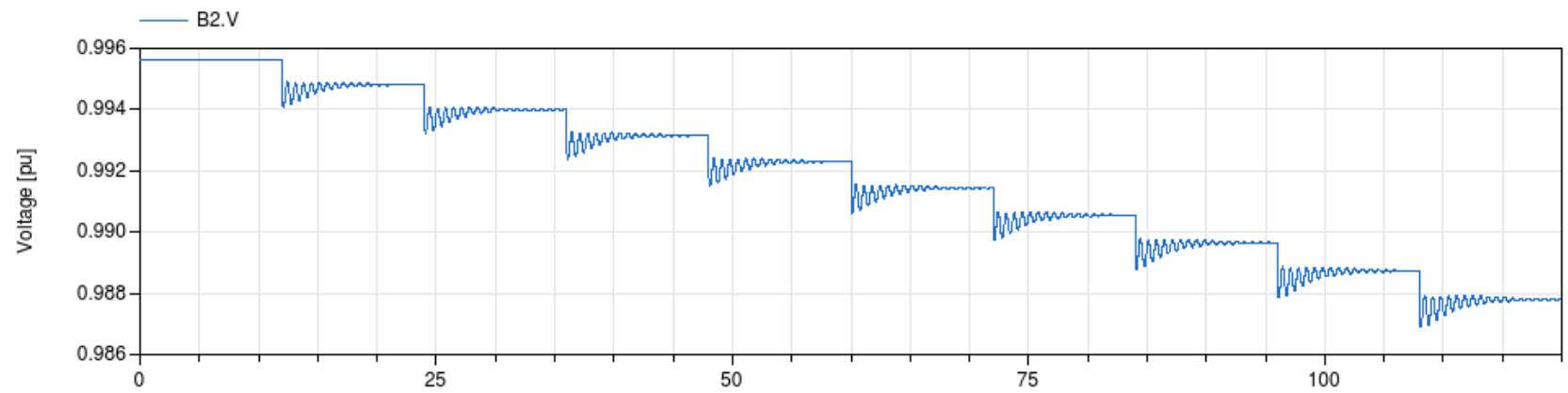

(a) Voltage in bus 02 for simulation I, distribution feeder in bus 02 .

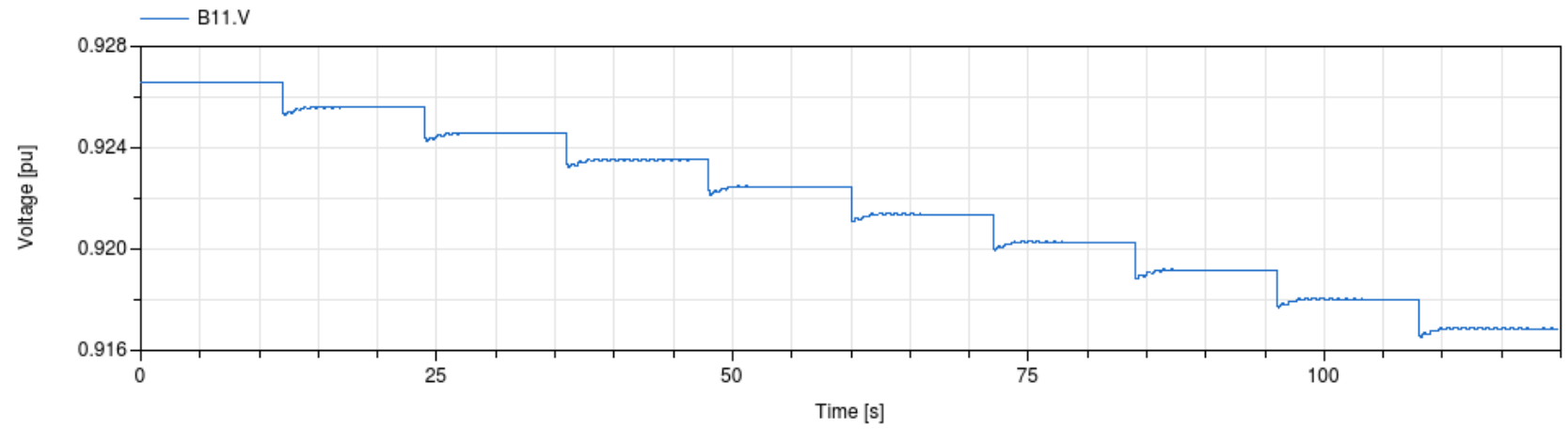

(b) Voltage in bus 11 for simulation IV, distribution feeder in bus 11 .

Figure 6. Voltage behavior in buses 02 and 11 during the 120 seconds of simulation.

Table 6. Comparison between eigenvalues of tests I and III.

\begin{tabular}{ccc}
\hline Load Step \# & $\begin{array}{c}\text { Error in } \\
\text { Damping }(\Delta \sigma)\end{array}$ & $\begin{array}{c}\text { Error in } \\
\text { Frequency }(\Delta \omega)\end{array}$ \\
\hline 1 & -0.00003 & 0.00003 \\
2 & -0.00010 & 0.00011 \\
3 & -0.00030 & 0.00027 \\
4 & -0.00059 & 0.00047 \\
5 & -0.00094 & 0.00076 \\
6 & -0.00143 & 0.00110 \\
7 & -0.00193 & 0.00156 \\
8 & -0.00258 & 0.00210 \\
9 & -0.00335 & 0.00273 \\
\hline
\end{tabular}

Table 7. Comparison between eigenvalues of tests IV and VI.

\begin{tabular}{cc}
\hline Load Step \# & Error in damping $(\Delta \sigma)$ \\
\hline 1 & -0.00002 \\
2 & -0.00009 \\
3 & -0.00021 \\
4 & -0.00038 \\
5 & -0.00062 \\
6 & -0.00093 \\
7 & -0.00133 \\
8 & -0.00183 \\
9 & -0.00245 \\
\hline
\end{tabular}

\section{Conclusions}

This paper presented mathematical modeling and implementation in Modelica language of three-phase models for 


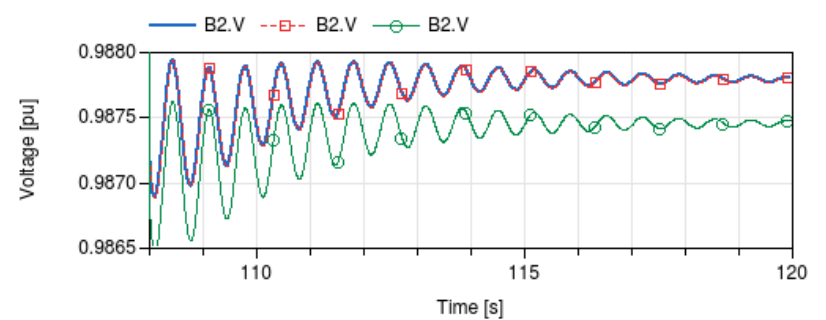

(a) Comparison between voltage in bus 02 for simulations I, II and III.

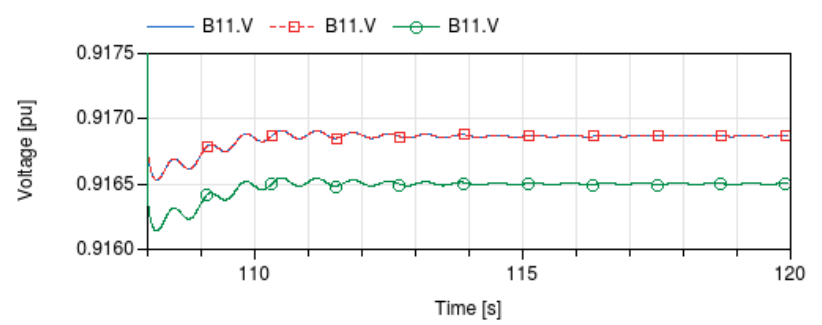

(b) Comparison between voltage in bus 11 for simulations IV, V and VI.

Figure 7. Voltages in buses 02 and 11 for corresponding simulation sets, during the last load step.

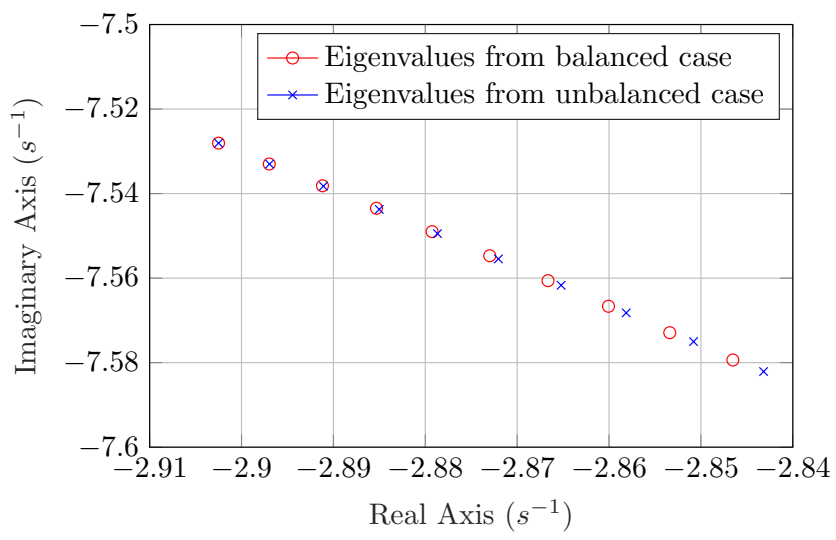

Figure 8. Comparison between specific eigenvalue coming from simulations I and III.

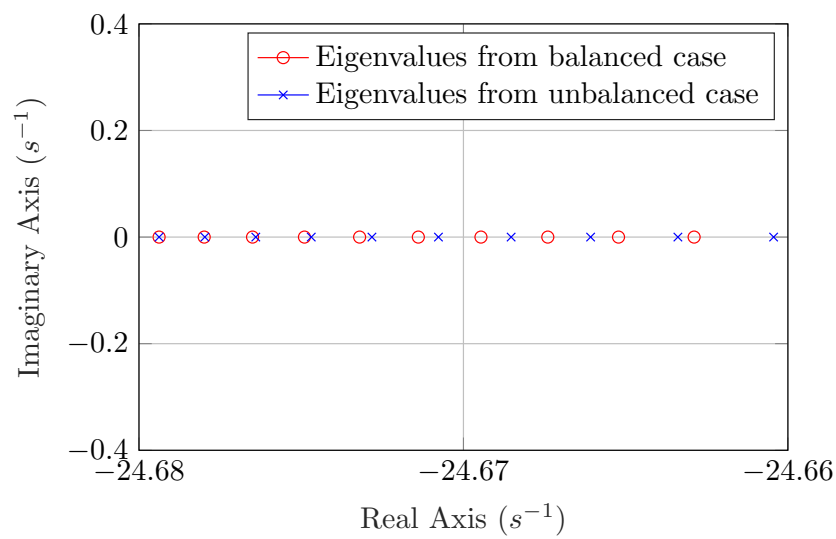

Figure 9. Comparison between specific eigenvalue coming from simulations IV and VI. transmission lines and a hybrid positive sequence threephase models for T\&D power networks. These models are used to represent a three-phase distribution feeder connected to benchmark IEEE 14-bus transmission system, modeled in its positive sequence equivalent. Six different simulations are realized to compare the different models for the distribution feeder (positive sequence versus threephase) and to study different conditions to load increase in two specific buses (balanced and unbalanced).

The results from dynamic simulations presented interesting and expected results. Voltage behavior observed using positive sequence models is the same one when the three-phase model had a balanced load increase. Furthermore, results coming from unbalanced load increase show that a positive sequence model might lead to erroneous results of the system dynamic behavior. This fact is corroborated by the linearization analysis performed in this paper. The comparison between eigenvalues coming from the linearized systems shows that, in fact, a positive sequence model for the distribution feeder could lead to wrong conclusions about system stability.

In addition, it is important to highlight that linear analysis is easily conducted in this paper using Modelicacompliant software. Software packages commonly used to study power systems do not necessarily have linear analyses tool, and are encoded separately. This analysis is important specifically to design controllers in the power system. Thus, in this specific matter, the Modelica language and compliant tools may assist in the development of new computational software tools to analyze complex power systems that should emerge in the near future.

The main results of this paper are particularly meaningful, as distributed generation at low-voltage levels is being introduced, especially in the form of photo-voltaic generation; which imminently will give rise to unbalances in distribution networks with single-phase feeders. This type of distribution networks are being referred to as "Active" Distribution Networks (ADNs), which are expected to increase in the near future. While the studies in this paper did not address ADNs specifically, the main result already highlights the importance of unbalances, and naturally, the addition of components with dynamics in distribution feeders will increase this impact. Hence, future work will analyze the impact on power system stability when modeling DG in distribution feeders, and in particular, the impact of their control response. In addition, the study of larger and more complex systems would be of great value and the authors consider it as a future study.

\section{Acknowledgements}

This work was supported in whole or in part by the Engineering Research Center Program of the National Science Foundation and the Department of Energy under Award EEC-1041877, and in part by the CURENT Industry Partnership Program. 


\section{References}

J. Arrillaga, C. P. Arnold, and B. J. Harker. Computer Modelling of Electrical Power Systems, volume 2. Wiley Online Library, 2001.

T. M. L. Assis, G. N. Taranto, D. M. Falcao, and A. Manzoni. Long and short-term dynamic simulations in distribution networks with the presence of distributed generation. In Power Engineering Society General Meeting, 2006. IEEE, pages 7pp. IEEE, 2006.

K. Balasubramaniam and S. Abhyankar. A combined transmission and distribution system co-simulation framework for assessing the impact of volt/var control on transmission system. In 2017 IEEE Power Energy Society General Meeting, pages 1-5, July 2017. doi:10.1109/PESGM.2017.8274633.

M. Baudette, M. Castro, T. Rabuzin, J. Lavenius, T. Bogodorova, and L. Vanfretti. OpenIPSL: Open-instance power system library—update 1.5 to "iTesla power systems library (iPSL): A modelica library for phasor time-domain simulations". SoftwareX, 7:34-36, 2018.

Jens C Boemer, Karsten Burges, Pavel Zolotarev, Joachim Lehner, Patrick Wajant, Markus Fürst, Rainer Brohm, and Thomas Kumm. Overview of german grid issues and retrofit of photovoltaic power plants in germany for the prevention of frequency stability problems in abnormal system conditions of the entso-e region continental europe. In 1st international workshop on integration of solar power into power systems, volume 24, 2011.

F. Casella, A. Bartolini, S. Pasquini, and L. Bonuglia. Objectoriented modelling and simulation of large-scale electrical power systems using modelica: A first feasibility study. In Industrial Electronics Society, IECON 2016-42nd Annual Conference of the IEEE, pages 6298-6304. IEEE, 2016.

F. Casella, A. Leva, and A. Bartolini. Simulation of large grids in OpenModelica: reflections and perspectives. In Proceedings of the 12th International Modelica Conference, Prague, Czech Republic, May 15-17, 2017, number 132, pages 227233. Linköping University Electronic Press, 2017.

Cepel. ANAREDE. https://bit. ly/2Mjkd1F, a.

Cepel. ANATEM. https://bit. Iy/2FzTKw4, b.

M. de Castro Fernandes, J. G. de Oliveira, L. Vanfretti, M. Baudette, and M. A. Tomim. Modeling and simulation of a hybrid single-phase/three-phase system in modelica. In 2018 Simposio Brasileiro de Sistemas Eletricos (SBSE), pages 1-7, May 2018. doi:10.1109/SBSE.2018.8395775.

EATON. Cymdist. https://bit. ly/2HkxHer.

ENTSO-E. Dispersed Generation Impact on CE Region Security. European Network of Transmission System Operators of Electricity, ENTSO-E, 2014.

EPRI. OpenDSS. https://bit. 1y/2zV4cLB.

P. A. N. Garcia, J. L. R. Pereira, S. Carneiro, V. M. da Costa, and N. Martins. Three-phase power flow calculations using the current injection method. IEEE Transactions on Power Systems, 15(2):508-514, 2000.
IEA. World Energy Outlook 2017. Organization for Economic Co-operation and Development, OECD, 2017.

H. Jain, K. Rahimi, A. Tbaileh, R. P. Broadwater, A. K. Jain, and M. Dilek. Integrated transmission \& distribution system modeling and analysis: Need \& advantages. In Power and Energy Society General Meeting (PESGM), 2016, pages 1-5. IEEE, 2016.

W. H. Kersting. Distribution System Modeling and Analysis. CRC press, 2001.

S. K. M. Kodsi and C. A. Canizares. Modeling and simulation of IEEE 14-bus system with FACTS controllers. University of Waterloo, Canada, Tech. Rep, 2003.

J. M. T. Marinho and G. N. Taranto. A hybrid three-phase singlephase power flow formulation. IEEE Transactions on Power Systems, 23(3):1063-1070, 2008.

F. Milano. An open source power system analysis toolbox. IEEE Transactions on Power Systems, 20(3):1199-1206, Aug 2005. ISSN 0885-8950. doi:10.1109/TPWRS.2005.851911.

M. Mirz, L. Netze, and A. Monti. A multi-level approach to power system modelica models. In Control and Modeling for Power Electronics (COMPEL), 2016 IEEE 17th Workshop on, pages 1-7. IEEE, 2016.

M. A. A. Murad, F. J. Gómez, and L. Vanfretti. Equation-based modeling of three-winding and regulating transformers using modelica. In 2015 IEEE Eindhoven PowerTech, pages 1-6, June 2015. doi:10.1109/PTC.2015.7232503.

Siemens. PSS/E software. https://sie.ag/2DpsTR9.

G. N. Taranto and J. M. T. Marinho. Simulation of integrated transmission and distribution networks with a hybrid threephase/single-phase formulation. Interface, 2, 2017.

W. F. Tinney and C. E. Hart. Power flow solution by newton's method. IEEE Transactions on Power Apparatus and systems, (11):1449-1460, 1967.

L. Vanfretti, W. Li, T. Bogodorova, and P. Panciatici. Unambiguous power system dynamic modeling and simulation using modelica tools. In 2013 IEEE Power Energy Society General Meeting, pages 1-5, July 2013. doi:10.1109/PESMG.2013.6672476.

Luigi Vanfretti, Tetiana Bogodorova, and Maxime Baudette. A modelica power system component library for model validation and parameter identification. In Proceedings of the 10th International Modelica Conference; Lund; Sweden, number 96, pages 1195-1203. Linköping University Electronic Press, 2014.

D. Winkler. Electrical power system modelling in modelicacomparing open-source library options. In Proceedings of the 58th Conference on Simulation and Modelling (SIMS 58) Reykjavik, Iceland, September 25th-27th, 2017, number 138, pages 263-270. Linköping University Electronic Press, 2017. 